\title{
BORONIZADO DE ALEACIONES DE BASE COBRE
}

\author{
Ernesto Ponce ${ }^{1} \quad$ Luis Martínez ${ }^{2} \quad$ José Vargas $^{2}$ \\ Recibido el 20 de julio de 2004, aceptado el 22 de noviembre de 2004
}

\begin{abstract}
RESUMEN
Se presenta el tratamiento térmico de algunas aleaciones de base Cobre empleando sales que contienen Boro. El objetivo principal es producir una capa de metal con mayor dureza y menor coeficiente de rozamiento que el proveniente de fábrica, para aplicaciones de piezas combinadas de acero- latón con movimiento relativo como cojinetes deslizantes, bujes, engranajes, levas y transmisiones sinfín corona, entre otros. La ventaja del método radica en que el Boro es un átomo pequeño comparado con otros elementos y difunde con facilidad en la superficie del metal base, formando compuestos. Pueden resultar capas muy definidas en composiciones mono o polifásicas, estas capas se caracterizan por tener alta microdureza, elevado punto de fusión y bajo coeficiente de roce, lo que contribuye a reducir el desgaste y tiene un mejor comportamiento tribológico.
\end{abstract}

Palabras claves: Boronizado, aleaciones base cobre.

\begin{abstract}
This paper shows the heat treatment of some copper alloys employing Boron salts. The main objective is to produce a stronger metal layer and smaller friction coeficient than the original alloy, for application on SteelBronze moving parts such as bearings, gears, cams, worm gears and slide mechanisms. The advantage of the method is based on the atom of Boron, smaller than the others, who easily diffusses in the surface of the base metal. Very well defined layers of mono or poly phases Boron compounds may result, having high melting point and low friction coefficient, thus reducing wear and exhibiting a better tribological performance.
\end{abstract}

Keywords: Boronizing, copper based alloys.

\section{INTRODUCCIÓN}

En el Norte de Chile existen yacimientos de ulexita, compuesto natural que contiene boratos. Su aplicación industrial es de una gama muy variada, tal como fabricación de fibras de vidrio, cristales, pantallas de televisores, cerámicas, elementos de control de radioactidad en centrales nucleares, agricultura y metalurgia, por mencionar algunas. Entre las aplicaciones metalúrgicas está el boronizado, que cubre un amplio campo en los materiales ferrosos, no ferrosos y cerámicos. En algunos casos se aplica con tecnologías complejas, como plasma o, gases y en otros, con técnicas al alcance de cualquier maestranza que disponga de un equipo mínimo para tratamientos térmicos.

A partir de minerales como la ulexita $\left(\mathrm{NaCaB}_{5} \mathrm{O}_{9}\right.$ $\left.8 \mathrm{H}_{2} \mathrm{O}\right)$, la colemanita $\left(\mathrm{Ca}_{2} \mathrm{~B}_{6} \mathrm{O}_{11} 5 \mathrm{H}_{2} \mathrm{O}\right)$, la kernita $\left(\mathrm{Na}_{2} \mathrm{~B}_{4} \mathrm{O}_{7} 4 \mathrm{H}_{2} \mathrm{O}\right)$ y la boracita $\left(\mathrm{Mg}_{7} \mathrm{C}_{12} \mathrm{~B}_{16} \mathrm{O}_{30}\right)$ se obtiene ácido bórico $\left(\mathrm{H}_{3} \mathrm{BO}_{4}\right)$ y bórax $\left(\mathrm{Na}_{2} \mathrm{~B}_{4} \mathrm{O}_{7}\right.$ $10 \mathrm{H}_{2} \mathrm{O}$ ), materia prima de la que se obtienen los productos industriales. Aunque el Boro por su posición en el sistema periódico indicaría una relación parecida al Aluminio, es mucho más parecido al Carbono y al Silicio en sus propiedades químicas. El Boro cristalino es similar al diamante en sus cualidades ópticas y apariencia, siendo casi tan duro como él. En sus compuestos actúa como un no metal pero en estado puro es un conductor eléctrico como el Carbono. Los hidruros de Boro son todavía más parecidos a los compuestos de Silicio y Carbono. Otros compuestos industriales de importancia son el carburo de Boro $\left(\mathrm{B}_{4} \mathrm{C}\right)$. El Boro tiene aplicaciones en el campo de la energía nuclear, por su alta capacidad para absorber neutrones se utiliza como controlador en los reactores nucleares y como constituyente de los escudos contra neutrones. Durante la Guerra Fría fue almacenado por miles de toneladas en la ex Unión Soviética, a fin de descontaminar los campos de cultivo y parte de

\footnotetext{
${ }^{1}$ Universidad de Tarapacá, 18 de septiembre 2222, Arica - Chile. eponce@uta.cl

${ }^{2}$ Alumnos memoristas de Ingeniería Mecánica
} 
ciudades en la eventualidad de un conflicto nuclear. Un trabajo anterior relacionado con aceros permitió solucionar algunos casos de alto desgaste y rozamiento, mediante sencillos procedimientos de tratamiento térmico[1]. Dado que nuestro país es uno de los principales productores de Cobre, tiene sentido el realizar experiencias con aleaciones de este metal. Actualmente se investigan y patentan métodos avanzados para mejorar aleaciones, [7], [8] y [10].

\section{METODOLOGÍA}

Se trataron barras comerciales de latón SAE 72 y SAE 73 de sección redonda, por derivarse de ellas las aplicaciones más comunes como cojinetes deslizantes. Se usaron 8 mezclas boronizantes, empleándose ácido bórico, ulexita, bórax, carbón vegetal, arcilla y arena. Las mezclas derivan de trabajos anteriores relacionados con tratamientos de bronce. La mejor elección se basó en la mayor microdureza y la mayor penetración de la capa superficial. Estas últimas experiencias se llevaron a cabo por microscopía óptica. La temperatura de trabajo se mantuvo en $850^{\circ} \mathrm{C}$ por un tiempo de 3,5 horas. Las mezclas de sales se efectuaron con material tamizado en malla de abertura $1,5 \mathrm{~mm}$, debido a que en pasadas experiencias un tamaño menor no arrojó buenos resultados. Se midió la dureza del latón en el estado en que provenía. Los valores de dureza así medidos corresponden a un endurecimiento por deformación. Los tratamientos se efectuaron en crisoles de cerámica debido a que los envases de acero absorbían una parte del Boro. Las piezas a tratar se recubrieron totalmente en la mezcla de sales, siendo esta compactada para evitar la entrada de aire, además cada crisol era cerrado con una tapa de cerámica fabricada con este propósito, bajo la pieza también se compactó una "cama" de sales, [9].

\section{DESARROLLO}

\section{Mezclas de Sales}

Las mezclas estuvieron formadas por la composición en peso mostrada en la Tabla 1.

Tabla 1. Composición de mezclas para tratamientos térmicos.

\begin{tabular}{|c|c|c|c|c|c|}
\hline MEZCLA & $\begin{array}{c}\mathrm{H}_{3} \mathrm{BO}_{3} \\
\%\end{array}$ & $\begin{array}{c}\text { CARBÓN } \\
\text { VEGETAL \% }\end{array}$ & $\begin{array}{c}\text { ARCILLA Y } \\
\text { ARENA \% }\end{array}$ & $\begin{array}{c}\mathbf{N A}_{2} \mathbf{B}_{4} \mathbf{O}_{7} \\
\%\end{array}$ & $\begin{array}{c}\text { ULEXITA } \\
\%\end{array}$ \\
\hline A & 20 & 30 & 45 & 5 & --- \\
\hline B & 10 & 85 & --- & 5 & --- \\
\hline $\mathrm{C}$ & 10 & 90 & --- & --- & --- \\
\hline D & --- & 70 & --- & --- & 30 \\
\hline E & --- & 90 & -- & -- & 10 \\
\hline $\mathrm{F}$ & 20 & 35 & 45 & --- & --- \\
\hline G & 20 & 80 & --- & --- & --- \\
\hline $\mathrm{H}$ & --- & 80 & --- & --- & 20 \\
\hline
\end{tabular}

\section{Medición del Rozamiento}

Se realizó con un equipo de laboratorio formado por un goniómetro y un plano giratorio donde se fijó una lámina pulida de acero. Este plano inclinado primeramente se dispone en posición horizontal con la pieza de latón en el extremo alejado de la articulación. Luego, con una manivela se le hace rotar lentamente merced a una transmisión. Cuando empieza a producirse el movimiento se detiene la rotación y se mide el ángulo. La tangente de este ángulo es el coeficiente de rozamiento seco entre el acero y el latón, [2].
Para ensayo húmedo con distintos lubricantes debe realizarse el ensayo de compresión de anillo de geometría $\mathrm{D}: \mathrm{d}: \mathrm{h}=6: 3: 2$, con ranuras en las caras planas para producir compensación de presiones, [3], [4] y [6]. Este trabajo no se hizo por escapar de los propósitos de este artículo.

\section{Probetas}

Se emplearon 9 probetas provenientes de varillas redondas estiradas de $10 \mathrm{~mm}$, se les dio diámetro = altura $=10 \mathrm{~mm}$. También se emplearon 3 probetas de 32 
$\mathrm{mm}$ en diámetro mecanizadas con altura $=10 \mathrm{~mm}$. Las pequeñas fueron montadas en resina y a las mayores después del tratamiento térmico se les pulimentó a pleno diámetro. Una de cada especie se dejó sin tratamiento térmico para comparar su dureza con las otras.

\section{Microdurezas}

Una vez realizado el tratamiento se realizan las mediciones desde la periferia hacia el núcleo. La carga empleada fue de $100 \mathrm{~g}$. Posteriormente se compara con las medidas en las probetas sin tratar y las durezas consideradas standard.

\section{Tiempo del Tratamiento}

En el presente trabajo se analizaron espesores de capa con una misma mezcla de sales. Como ya se sabía que existe un tiempo óptimo mas allá del cual la profundidad de la capa se incrementa cada vez menos, se entregan dos resultados que demuestran lo dicho: para 3,5 h el espesor fue de $0,22 \mathrm{~mm}$ y para 6 horas sólo ascendió a $0,28 \mathrm{~mm}$. El tiempo de 3,5 h había resultado ideal en otros casos, [1].

\section{Composición Química de los Latones}

Se analizaron por fluorescencia de rayos $\mathrm{X}$ el llamado latón de corte SAE 72 y el designado como latón naval SAE 73, ambos producidos por la empresa nacional SORENA. Los análisis fueron realizados antes del tratamiento térmico y se muestran en la Tabla 2.

\section{Dureza de las Probetas}

Antes del tratamiento térmico se midió la dureza media de las probetas. En el SAE 72 los porcentajes de Zn están entre el 20 y el $36 \%$, lo que significaría un metal más dúctil que el naval. Para el SAE 73 el Zn está sobre el $36 \%$, luego será mas duro que el anterior, [5].Esto se indica en la Tabla 3.

Tabla 2. Porcentaje de elementos presentes en latones tratados.

\begin{tabular}{|c|c|c|}
\hline ELEMENTO & $\begin{array}{c}\text { SAE 72 \% } \\
\text { CONCENTRACIÓN }\end{array}$ & $\begin{array}{c}\text { SAE 73 \% } \\
\text { CONCENTRACIÓN }\end{array}$ \\
\hline $\mathrm{Cu}$ & 57,90 & 57,33 \\
$\mathrm{Zn}$ & 35,70 & 40,40 \\
$\mathrm{~Pb}$ & 2,06 & 1,15 \\
$\mathrm{Si}$ & 1,53 & 0,28 \\
$\mathrm{Cl}$ & 0,94 & 0,09 \\
$\mathrm{Al}$ & 0,79 & --- \\
$\mathrm{S}$ & 0,51 & 0,04 \\
$\mathrm{Ca}$ & 0,23 & 0,03 \\
$\mathrm{Fe}$ & 0,15 & 0,15 \\
$\mathrm{Sn}$ & 0,11 & 0,41 \\
$\mathrm{~K}$ & 0,08 & --- \\
$\mathrm{Ni}$ & --- & 0,12 \\
\hline
\end{tabular}

Tabla 3. Durezas medidas vs las indicadas en la literatura.

\begin{tabular}{|c|c|c|}
\hline $\begin{array}{c}\text { ALEACIÓN } \\
\text { DE COBRE }\end{array}$ & $\begin{array}{c}\text { DUREZA INDICADA HV } \\
\text { POR LA LITERATURA }\end{array}$ & $\begin{array}{c}\text { DUREZA MEDIA } \\
\text { HV MEDIDA }\end{array}$ \\
\hline SAE 72 & 100 & 82 \\
SAE 73 & 100 & 112 \\
\hline
\end{tabular}

\section{RESULTADOS}

\section{Micrografías}

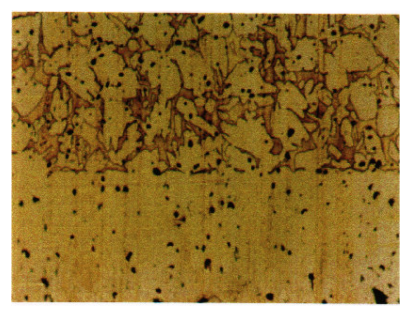

Fig. 1 X 100. Latón SAE 72. Mezcla B. Espesor capa $=0.21 \mathrm{~mm} .162,5 \mathrm{HV}$. Zona superficial.

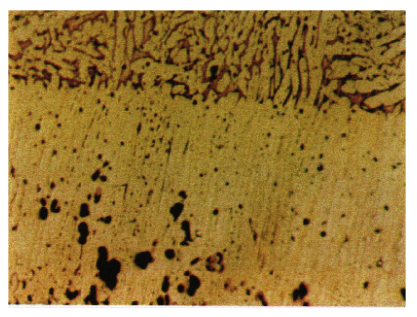

Fig. 2 X 100. Latón
Mezcla A. Espesor capa $=0.35 \mathrm{~mm}$. $206 \mathrm{HV}$. Zona superficial.

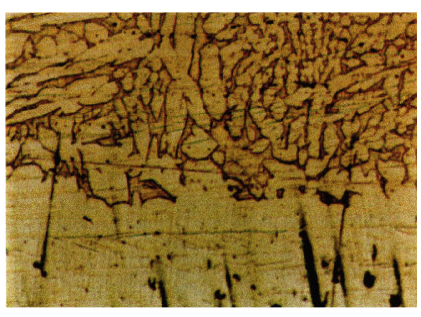

Fig. 3 X 100. Latón SAE 73. Zona superficial. Espesor capa $=0.18 \mathrm{~mm}$. Mezcla B. 143,1 HV.

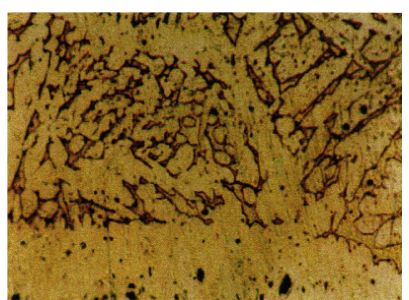

Fig. 4 X 100. Latón SAE 73. Zona superficial.

Espesor de capa $=0.15 \mathrm{~mm}$. Mezcla A. 


\section{Latón SAE 72 Boronizado}

El tratamiento termoquímico se realizó en 8 probetas con mezcla de sales ya identificadas en la Tabla 1 . Temperatura: 850 grados C; tiempo 3,5 h. Las Tablas 4 y 5 entregan los resultados.

\section{Latón SAE 73 Boronizado}

Con las dos mejores mezclas boronizantes que dieron mayor dureza de capa hasta $0,2 \mathrm{~mm}$ (la A y B) se realizó el tratamiento térmico a dos probetas de latón SAE 73 de diámetro $32 \mathrm{~mm}$ y altura $10 \mathrm{~mm}$. Las piezas fueron cortadas longitudinalmente para apreciar mejor la capa y la difusión hacia el núcleo. Los resultados aparecen en las Tablas 6 y 7 . Temperatura $850^{\circ} \mathrm{C}$ durante $3.5 \mathrm{~h}$.

Tabla 4. Microdurezas en latones SAE 72 tratados térmicamente.

\begin{tabular}{|c|c|c|c|c|c|c|c|c|}
\hline \multirow{2}{*}{$\begin{array}{c}\text { Distancia } \\
\text { radial desde } \\
\text { superficie } \\
(\mathbf{m m})\end{array}$} & \multicolumn{8}{|c|}{$\begin{array}{l}\text { MICRODUREZA VICKERS (Kp/mm2) } \\
\text { MEZCLAS BORONIZANTES }\end{array}$} \\
\hline & $\mathrm{A}$ & $\bar{B}$ & $\mathrm{C}$ & $\mathrm{D}$ & $\bar{E}$ & $\bar{F}$ & $\mathrm{G}$ & $\mathrm{H}$ \\
\hline 0,1 & 147,1 & 151,4 & 128,4 & 125,1 & 107,7 & 59,1 & 102,7 & 53,3 \\
\hline 0,2 & 206 & 165,2 & 125,1 & 115,9 & 110,3 & 93,6 & 100,3 & 77,2 \\
\hline 0,3 & 186,9 & 170,3 & 139,2 & 135,5 & 121,9 & 95,79 & 87,6 & 89,6 \\
\hline 0,4 & 181,1 & 170,3 & 139,2 & 151,4 & 128,4 & 118,9 & 91,6 & 84 \\
\hline 0,5 & 181,1 & 175,6 & 143,1 & 135,5 & 135,5 & 128,4 & 105,1 & 91,6 \\
\hline 0,6 & 193 & 170,3 & 151,4 & 139,1 & 143,1 & 125,1 & 102,7 & 93,6 \\
\hline 0,7 & 186,9 & 143,1 & 151,4 & 143,1 & 115,9 & 118,9 & 95,8 & 74,2 \\
\hline 0,8 & 186,9 & 151,4 & 151,4 & 128,4 & 147,1 & 118,9 & 143,1 & 93,6 \\
\hline 0,9 & 199,3 & 151,4 & 139,2 & 147,1 & 115,9 & 125,1 & 67,3 & 74,2 \\
\hline 1 & 199,3 & 151,4 & 139,2 & 135,5 & 128,4 & 121,9 & 95,8 & 82,2 \\
\hline 2 & 143,1 & 147,1 & 147,2 & 147 & 151,4 & 139,2 & 98 & 82,2 \\
\hline 3 & 118,9 & 147 & 151,4 & 135,5 & 135 & 128,4 & 107,7 & 91,6 \\
\hline 4 & 135,5 & 143,1 & 131,9 & 143,1 & 170.3 & 125,1 & 102,7 & 75,7 \\
\hline 5 & 139,2 & 151,4 & 131,9 & 170,3 & 135,5 & 131,9 & 121,9 & 85,8 \\
\hline
\end{tabular}

Tabla 5. Espesor de capa en SAE 72.

\begin{tabular}{|c|c|}
\hline Mezcla & $\begin{array}{c}\text { Espesor Capa } \\
\text { Superficial } \\
\text { Visible (mm) }\end{array}$ \\
\hline A & 0,35 \\
B & 0,21 \\
C & 0,19 \\
D & 0,12 \\
E & 0,18 \\
F & 0,25 \\
G & 0,23 \\
H & 0,22 \\
\hline
\end{tabular}

Tabla 6. Microdurezas en mezclas a y b de latones SAE 73 tratados térmicamente.

\begin{tabular}{|c|c|c|c|c|c|}
\hline $\begin{array}{c}\text { Distancia } \\
\text { radial desde } \\
\text { superficie } \\
(\mathbf{m m})\end{array}$ & \multicolumn{2}{|c|}{$\begin{array}{c}\text { MICRODUREZA } \\
\text { VICKERS (Kp/mm2) }\end{array}$} & $\begin{array}{c}\text { Distancia } \\
\text { radial desde } \\
\text { Superficie }\end{array}$ & \multicolumn{2}{|c|}{$\begin{array}{c}\text { MICRODUREZA } \\
\text { VICKERS (Kp/mm2) }\end{array}$} \\
\cline { 2 - 6 } & $\mathrm{A}$ & $\mathrm{B}$ & $(\mathrm{mm})$ & $\mathrm{A}$ & $\mathrm{B}$ \\
\hline 0,1 & 51,5 & 135,5 & 5 & 165,2 & 206 \\
\hline 0,2 & 66 & 143,1 & 6 & 165,2 & 206 \\
\hline 0,3 & 107,7 & 128,4 & 7 & 151,4 & 220,5 \\
\hline 0,4 & 110,3 & 135,5 & 8 & 165,2 & 193 \\
\hline 0,5 & 115,9 & 151,4 & 9 & 155,8 & 170,3 \\
\hline 0,6 & 139,2 & 151,4 & 10 & 155,8 & 193 \\
\hline 0,7 & 95,8 & 135,5 & 11 & 125,1 & 206 \\
\hline 0,8 & 121,9 & 135,5 & 12 & 143,1 & 236,5 \\
\hline 0,9 & 115,9 & 170,3 & 13 & 143,1 & 206 \\
\hline 1 & 100,3 & 151 & 14 & 175,6 & 206 \\
\hline 2 & 113,3 & 160,4 & 15 & 193,0 & 236,5 \\
\hline 3 & 128,4 & 170,3 & 16 & 213,1 & 206 \\
\hline 4 & 131,9 & 193 & & & \\
\hline
\end{tabular}

A y B son mezclas Boronizantes.

Revista Facultad de Ingeniería, Chile, Vol.12 N², 2004 
Tabla 7. Espesor de capa en SAE $73 \mathrm{~mm}$.

\begin{tabular}{|c|c|}
\hline Mezcla & $\begin{array}{c}\text { Espesor Capa Superficial } \\
\text { Visible }\end{array}$ \\
\hline A & 0,15 \\
B & 0,18 \\
\hline
\end{tabular}

Tabla 8. Coeficientes de rozamiento latones boronizados - acero.

\begin{tabular}{|l|l|l|}
\hline \multicolumn{1}{|c|}{ Latón } & \multicolumn{1}{|c|}{ Mezcla } & Coef. Rozamiento \\
\hline SAE 72 & A & 0,30 \\
SAE 72 & sin tratar & 0,40 \\
SAE 73 & B & 0,32 \\
SAE 73 & sin tratar & 0,41 \\
\hline
\end{tabular}

\section{CONCLUSIONES}

Con respecto al latón SAE 72, la mayor microdureza de capa fue obtenida con la mezcla A, con un valor de 206 $\mathrm{HV}$, que es más del doble del indicado por la probeta muestra ( $82 \mathrm{HV}$ ). La segunda fue obtenida con mezcla B, con 175,6 HV (ambas contenían un $5 \%$ de bórax). Como comparación, en acero AISI 1020 se obtiene una dureza de $160 \mathrm{HV}$ con un temple en aceite y revenido a $149{ }^{\circ} \mathrm{C}$. Debió ponerse mas atención en la mezcla D que indicó 170,3 HV en el núcleo, pero sólo 125,1 HV en la superficie. Esto lleva a pensar que difundió muy bien y si el tiempo en el horno hubiese sido menor que $3,5 \mathrm{~h}$, pudo haberse logrado mayor microdureza superficial. Las mezclas C, D, E, F, G presentan mejores características superficiales que la muestra, sin embargo, la $\mathrm{H}$ es la única que tiene un valor inferior, pero sólo en la superficie, puede atribuirse esta disminución a que sólo se empleó ulexita, no se incluyó ácido bórico ni bórax ni arcilla con sílice. Las mezclas A y $\mathrm{F}$ son parecidas en $\%$, pero no así en resultados, la diferencia mayor está en que la $\mathrm{F}$ carece del $5 \%$ de bórax, esto es suficiente para producir otro efecto. Todos los tratamientos generaron una capa, en mayor o menor grado, lo que prueba el fenómeno de difusión en el latón tratado, pero las combinaciones A, F, G, con un $20 \%$ de ácido bórico mostraron un espesor de capa mas pronunciado. El estudio contempló 8 mezclas de sales, cuyas concentraciones habían dado buenos resultados con $\mathrm{Cu}$ y otro bronce, pero sin duda habría sido deseable un mayor número de experiencias basadas en mayor información estadística. Los ensayos de rozamiento demuestran que hay una reducción significativa en su coeficiente de 0,40 de la pieza sin tratar a 0,30 en la boronizada, lo que es importante para algunos elementos de máquinas.

Con referencia al latón $\operatorname{SAE} 73$, lo que fue la mejor mezcla para el latón SAE 72 (mezcla A), no lo fue en absoluto para el SAE 73. Aquí según la Tabla 8, los resultados superiores en espesor de capa, microdureza superficial y del núcleo se obtuvieron con la mezcla B. Con el tratamiento el coeficiente de rozamiento bajó de 0,41 de la pieza sin tratar a 0,32 de la pieza boronizada.

\section{Comparación Entre los Tratamientos de Ambas Aleaciones}

Los efectos entre los dos latones demuestran resultados superiores con el latón SAE 72 frente al SAE 73. El mayor $\%$ de $\mathrm{Zn}$ en este último pudo originar la diferencia, tal como ocurrió con los aceros de alta aleación frente a los de baja, [1]. La experiencia debió además incluir la misma cantidad y tipo de sales que con el SAE 72 y con probetas de iguales dimensiones. Se hizo de esta manera para ganar tiempo y analizar en forma rápida un buen tratamiento para cojinetes con mayores dimensiones. Se hace notar que el mejor resultado para el latón naval no puede deducirse de este trabajo. Las mayores durezas crecientes hacia el interior de las piezas demuestran que fue excesivo el tiempo del tratamiento, mejores resultados superficiales se habrían logrado con tiempos menores. Pese a lo modesto del trabajo presentado y a sus indudables limitaciones, se concluye que con medios económicos reducidos puede darse un tratamiento térmico de buenas características a latones comerciales fabricados en el país.

\section{COMENTARIOS}

Un estudio más exhaustivo requiere realizar mayor número de análisis incorporando otras mezclas, buscando tiempos y temperaturas de difusión más eficientes. Estudios más acabados del rozamiento requieren trabajar con más parámetros, como rugosidad superficial para distintas interfases, lubricación y temperatura, entre otros. Para ello debe realizarse el ensayo de compresión de anillo y contar con una máquina de ensayos de gran capacidad de carga. Para conocer las nuevas características adquiridas de los materiales tratados, deberían hacerse ensayos de fatiga, resiliencia, tracción y cizalle. 


\section{REFERENCIAS}

[1] E.Ponce y otros; "Tratamientos Térmicos Experimentales con Boro". Anales Congreso Nacional de Ingeniería Mecánica. Valdivia, 1996.

[2] A.Higdon, W.Stiles; "Mecánica Técnica". Ed. Aguilar. Madrid, 1965.

[3] L.Herrera; “Análisis Experimental y Modelización de Procedimientos para Determinar las Características de Fluencia Plástica de los Materiales Dúctiles". Tesis Doctoral ETSII, UPM, 1984.

[4] N.Abdul; "Assessment for the lubrication for the cold extrusion of steel "; Machine Tool Design and Research Conference, Num.17 ,1977, pp. 401-409.

[5] American Society for Metals: Metal Handbook, Metallography, Structures and Phase Diagrams"; $8^{\circ}$ edition ,Ed. Metals Park, Vol. VIII,1973.
[6] V.Nagpal, D.Lahoti and T.Altan; "A numerical Method for simultaneous Prediction of Metal Flow and Temperatures in Upset Forging of Rings". Journal of Engineering for Industry, Vol.100 Nov. 1998 pp. 413-419.

[7] Kumar, Anil et al.; "Boriding". Bohn P.D., pp 437-447, 1989.

[8] K.Matiassovsky, et al.; "Boronizing Coating", Surface Coating Technology, vol.35 pp. 133-119, 1988.

[9] P.Molera; "Tratamientos Térmicos de los Metales"; Ed. Marcombo, España, 1991.

[10] US6783794; "Method and Arrangement for Plasma Boronizing"; Patentado el 31/08/2004 en USA. 